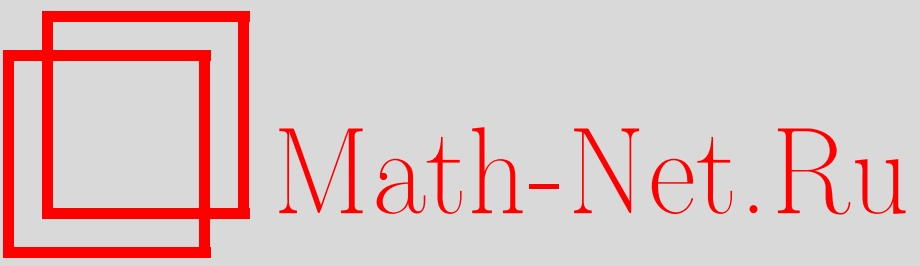

Г. И. Ивченко, Ю. И. Медведев, В. А. Миронова, Анализ спектра случайных симметрических булевых функций, Матем. вопр. криптогр., 2013, том 4, выпуск $1,59-76$

DOI: https://doi.org/10.4213/mvk73

Использование Общероссийского математического портала Math-Net.Ru подразумевает, что вы прочитали и согласны с пользовательским соглашением

http: //www . mathnet.ru/rus/agreement

Параметры загрузки:

IP : 54.147 .182 .235

26 апреля 2023 г., 10:57:45 
УДК 519.212.2

\title{
Анализ спектра случайных симметрических булевых функций
}

\author{
Г. И. Ивченко ${ }^{1}$, Ю. И. Медведев ${ }^{2}$, В. А. Миронова ${ }^{3}$ \\ ${ }_{1,3}$ НИУ Высшая школа экономики, Москва \\ ${ }^{2}$ Академия криптографии Российской Федерации, Москва
}

Получено 20.IV.2012

Исследуются различные вероятностные свойства спектра Уолша случайной симметрической булевой функции в общей стохастической модели, определяемой заданием на множестве всех симметрических функций некоторой вероятностной меры. В рассматриваемой модели ключевую роль играют свойства многочленов Кравчука, поэтому в работе приводится также сводка основных сведений о них.

Ключевые слова: симметрическая булева функция, преобразование Уолша, спектр функции, характеристическая функция, параметрическая мера, многочлены Кравчука, матрица Кравчука, спектральные характеристики, предельные теоремы

\section{Analysis of the spectrum of random symmetric Boolean functions}

G. I. Ivchenko ${ }^{1}$, Yu. I. Medvedev ${ }^{2}$, V. A. Mironova ${ }^{3}$

${ }^{1,3}$ NRU Higher School of Economics, Moscow

${ }^{2}$ Academy of Cryptography of the Russian Federation, Moscow

\begin{abstract}
General probabilistic model of a random symmetric Boolean function of $n$ variables is proposed. The characteristic function of the Walsh spectrum of a random symmetric Boolean function is defined; exact and asymptotic distributions of some spectrum characteristics as $n \rightarrow \infty$ are obtained in the case of the parametric measure. The basic properties of the Krawtchouk's polynomials (which are used in proofs) are reviewed.
\end{abstract}

Key words: symmetric Boolean function, Walsh transform, spectrum of function, characteristic function, parametric measure, Krawtchouk's polynomials, Krawtchouk matrix, spectrum characteristics, limit theorems

Citation: Mathematical Aspects of Cryptography, 2013, vol. 4, no. 1, pp. 59-88 (Russian). 
1. Введение: спектр симметрической булевой функции и вероятностная модель. Пусть $S_{n}$ - множество симметрических булевых функций от $n$ переменных $f(\bar{x}), \bar{x}=\left(x_{1}, x_{2}, \ldots, x_{n}\right) \in V_{n}=\{0,1\}^{n}$, то есть таких функций, которые принимают одно и то же значение на всех векторах $\bar{x}$ одинакового веса $\|\bar{x}\|=\sum_{i=1}^{n} x_{i}$. Симметрические булевы функции находят широкое применение в теории кодирования и криптографии, и их анализу посвящёно значительное число работ. Обзор публикаций на эту тему можно найти в статьях А. Канто и М. Видо [1], С. Саркара и С. Майтры [2] и М. Муффрона [3].

Обозначим $f(\bar{x})=f_{k}$, если $\|\bar{x}\|=k, k=0,1, \ldots, n$. Тогда вместо таблицы истинности функции $f$ (вектора размерности $2^{n}$ ) можно использовать более экономное её представление в виде двоичного вектора $\bar{f}_{n}=\left(f_{0}, f_{1}, \ldots, f_{n}\right)$ (номера единичных координат этого вектора называют иногда рабочими величинами, а нулевых - нерабочими).

Рассмотрим спектр Уолиа (далее - просто спектр) произвольной такой функции:

$$
w^{f}(\bar{u})=\sum_{\bar{x} \in V_{n}} f(\bar{x})(-1)^{(\bar{u}, \bar{x})}, \bar{u} \in V_{n} .
$$

Здесь, напомним, скалярное произведение $(\bar{u}, \bar{x})$ векторов пространства $V_{n}$ вычисляется по формуле $(\bar{u}, \bar{x})=\sum_{i=1}^{n} u_{i} x_{i}(\bmod 2)$.

Если $\|\bar{u}\|=m$, то соотношение (1) можно переписать в виде

$$
w^{f}(\bar{u})=\sum_{k=0}^{n} f_{k} \sum_{\bar{x}:|\bar{x}| \mid=k}(-1)^{(\bar{u}, \bar{x})}=\sum_{k=0}^{n} f_{k}\left[N_{0}(m, k ; n)-N_{1}(m, k ; n)\right],
$$

где

$$
N_{i}(m, k ; n)=|\{\bar{x}:\|\bar{x}\|=k,\|\bar{u}\|=m,(\bar{u}, \bar{x})=i\}|, \quad i=0,1
$$

Из (2) следует, что функция $w^{f}(\bar{u}), \bar{u} \in V_{n}$, также является симметрической, поэтому, обозначив $w^{f}(\bar{u})=w_{m}^{f}$, если $\|\bar{u}\|=m$, будем далее под спектром симметрической функции $f$ понимать вектор

$$
\bar{w}_{n}^{f}=\left(w_{0}^{f}, w_{1}^{f}, \ldots, w_{n}^{f}\right) .
$$


Далее для определённых в (3) величин нетрудно записать их явные выражения в виде следующих комбинаторных сумм:

$$
\begin{aligned}
& N_{0}(m, k ; n)=\sum_{j}\left(\begin{array}{l}
m \\
2 j
\end{array}\right)\left(\begin{array}{c}
n-m \\
k-2 j
\end{array}\right), \\
& N_{1}(m, k ; n)=\sum_{j}\left(\begin{array}{c}
m \\
2 j+1
\end{array}\right)\left(\begin{array}{c}
n-m \\
k-2 j-1
\end{array}\right),
\end{aligned}
$$

а их разность в (2) можно записать в виде

$$
\sum_{j}(-1)^{j}\left(\begin{array}{c}
m \\
j
\end{array}\right)\left(\begin{array}{c}
n-m \\
k-j
\end{array}\right)=P_{k}(m ; n),
$$

где $P_{k}(m ; n)$ - известные в анализе многочлены Кравчука [1], [2], [4].

В итоге спектральные коэффициенты $w_{m}^{f}$ симметрической функции $f$ можно записать в виде

$$
w_{m}^{f}=\sum_{k=0}^{n} f_{k} P_{k}(m ; n), \quad m=0,1, \ldots, n
$$

Если ввести матрииу Кравчука

$$
P_{n}=\left(P_{k}(m ; n)\right)_{k, m=0,1, \ldots, n},
$$

то соотношения (6) можно записать в матричном виде:

$$
\bar{w}_{n}^{f}=\bar{f}_{n} P_{n} .
$$

Это соотношение можно обратить. Действительно, как известно, булева функция однозначно определяется своим спектром (1) по формуле обращения

$$
f(\bar{x})=2^{-n} \sum_{\bar{u} \in V_{n}} w^{f}(\bar{u})(-1)^{(\bar{u}, \bar{x})}, \bar{x} \in V_{n},
$$

что для симметрических функций, аналогично выводу соотношения (6), можно переписать в виде

$$
f_{k}=2^{-n} \sum_{m=0}^{n} w_{m}^{f} P_{m}(k ; n), k=0,1, \ldots, n
$$


или в матричном виде

$$
\bar{f}_{n}=2^{-n} \bar{w}_{n}^{f} P_{n} .
$$

Из (8) и (9) следует, что матрица Кравчука невырождена и что обратная к ней матрица имеет вид

$$
P_{n}^{-1}=2^{-n} P_{n} .
$$

Отметим также следующее из (10) равенство

$$
P_{n}^{2}=2^{n} I_{n+1},
$$

где $I_{m}$ - единичная матрица порядка $m$.

Из (11) сразу следует известное соотношение ортогональности для многочленов Кравчука:

$$
\sum_{m=0}^{n} P_{k}(m ; n) P_{m}(r ; n)=2^{n} \delta_{k r},
$$

где $\delta_{k r}$ - символ Кронекера.

Дальнейшие свойства многочленов Кравчука будут представлены в следующем разделе.

Мы будем исследовать вероятностные свойства спектра $\bar{w}_{n}^{f}=\left(w_{0}^{f}, w_{1}^{f}, \ldots, w_{n}^{f}\right)$ случайной симметрической булевой функции $f$ в общей стохастической модели, когда на множестве $S_{n}$ задаётся некоторая вероятностная мера $\mathbf{P}$, приписывающая каждому вектору $\bar{f}_{n}=\left(f_{0}, f_{1}, \ldots, f_{n}\right)$ вес $\mathbf{P}\left(\bar{f}_{n}\right)$. Пусть

$$
\phi_{n}(\bar{t})=\mathbf{E} e^{i \bar{t} \bar{f}_{n}^{\prime}}, \bar{t}=\left(t_{0}, t_{1}, \ldots, t_{n}\right),
$$

есть соответствующая характеристическая функция.

Тогда характеристическая функция спектра $\bar{w}_{n}^{f}=\left(w_{0}^{f}, w_{1}^{f}, \ldots, w_{n}^{f}\right)$ с учётом (8) будет иметь вид

$$
\begin{aligned}
& \Phi_{n}(\bar{t})=\mathbf{E} e^{i \bar{t} \bar{w}_{n}^{\prime}}=\mathbf{E} e^{i\left(\bar{t} P_{n}^{\prime}\right) \bar{f}_{n}^{\prime}}=\phi_{n}\left(\bar{t} P_{n}^{\prime}\right)= \\
& =\phi_{n}\left(\sum_{m=0}^{n} t_{m} P_{k}(m ; n), k=0,1, \ldots, n\right) .
\end{aligned}
$$

В данной работе мы будем детально анализировать случай, когда компоненты вектора $\bar{f}_{n}=\left(f_{0}, f_{1}, \ldots, f_{n}\right)$ независимы и компонента $f_{k}$ принимает 
значения 0 и 1 с вероятностями соответственно $q_{k}$ и $p_{k}, p_{k}+q_{k}=1$ $(k=0,1, \ldots, n)$. Такую модель мы будем называть для краткости $\bar{p}$-моделью; в случае, когда все $p_{k}=p>0$, мы будем говорить о $p$-модели.

Для $\bar{p}$-модели формулы (13) и (14) принимают соответственно вид

$$
\phi_{n}(\bar{t})=\prod_{k=0}^{n} \mathbf{E} e^{i t_{k} f_{k}}=\prod_{k=0}^{n}\left(q_{k}+p_{k} e^{i t_{k}}\right)
$$

и

$$
\Phi_{n}(\bar{t})=\prod_{k=0}^{n}\left(q_{k}+p_{k} \exp \left\{i \sum_{m=0}^{n} t_{m} P_{k}(m ; n)\right\}\right) .
$$

Представление (15) является основой для дальнейшего анализа. Из него, а также из (8) видно, что в рассматриваемой модели ключевую роль играют свойства многочленов Кравчука, поэтому для удобства последующего изложения мы приводим в п. 2 сводку основных сведений о них.

2. Многочлены Кравчука и их свойства. Многочлены Кравчука были предложены в 30-х годах прошлого столетия украинским математиком М. Ф. Кравчуком, и они являются частным случаем обширного класса ортогональных многочленов дискретного переменного. Эти многочлены широко используются в различных задачах анализа и теории вероятностей, и они рассматривались в значительном числе работ. Это - монографии авторов Г. Сеге [4], Ф. Дж. Мак-Вильямса и Н. Дж. Слоэна [5], В. М. Сидельникова [6], Г. Бэйтмена, А. Эрдейи [7], В. Гузика и Р. Станицы [8], а также работы Р. Савицкого [9], В. И. Хохлова [10], [11] и других авторов. Из многочисленных свойств многочленов Кравчука здесь мы приводим лишь необходимые для последующего развития темы.

1) Из (5) легко находим вид производящей функции:

$$
P(z ; m, n)=\sum_{k} P_{k}(m ; n) z^{k}=(1-z)^{m}(1+z)^{n-m} .
$$

Используя обозначение $\left[z^{n}\right] f(z)=\operatorname{coef}_{z^{n}} f(z)$, можно записать, что

$$
P_{k}(m ; n)=\left[z^{k}\right](1-z)^{m}(1+z)^{n-m} .
$$

Замечание. В литературе можно встретить и другие, несколько отличные, определения многочленов Кравчука, например

$$
P_{k}(m ; n)=\left[z^{k}\right](1-z)^{m}(1+(l-1) z)^{n-m},
$$

где $l-$ дополнительный параметр (в нашем случае $l=2$ ). 
Из (16), в частности, следует, что

$$
\begin{gathered}
\sum_{k} P_{k}(m ; n)=0 \text { при } m>0, \\
P_{k}(0 ; n)=\left(\begin{array}{l}
n \\
k
\end{array}\right), P_{k}(n ; n)=(-1)^{k}\left(\begin{array}{l}
n \\
k
\end{array}\right) \text {, а также } P_{k}(m ; n)=(-1)^{k} P_{k}(n-m ; n) .
\end{gathered}
$$

Кроме того, так как при $m>0$

$$
(1-z)^{m-1}(1+z)^{n-m}=(1-z)^{-1} P(z ; m, n)=\sum_{N}\left(\sum_{k \leq N} P_{k}(m ; n)\right) z^{N},
$$

то, с учётом (16' ) и (17), имеем также следующие соотношения:

$$
\sum_{k \leq N} P_{k}(m ; n)=-\sum_{k>N} P_{k}(m ; n)=P_{N}(m-1 ; n-1) .
$$

Замечание. Целый класс линейных соотношений типа свёртки для многочленов Кравчука можно получить по следующей схеме: домножить левую и правую части соотношения (16) на $(1-z)^{ \pm r}$ либо на $(1+z)^{ \pm r}$ и разложить функцию в левой части по степеням $z$. Например, с учётом (16') получаем

$$
\begin{aligned}
& {\left[z^{k}\right](1-z)^{-r} P(z ; m, n)=\sum_{j=0}^{k}\left(\begin{array}{c}
r+j-1 \\
j
\end{array}\right) P_{k-j}(m ; n)=} \\
& =\left[z^{k}\right](1-z)^{m-r}(1+z)^{n-m}=P_{k}(m-r ; n-r) .
\end{aligned}
$$

Далее, из (16) имеем

$$
\begin{gathered}
\sum_{m} P(z ; m, n)\left(\begin{array}{l}
n \\
m
\end{array}\right) t^{m}=(1+z+t(1-z))^{n}=(1+t+z(1-t))^{n}= \\
=\sum_{k}\left(\begin{array}{l}
n \\
k
\end{array}\right) z^{k}(1-t)^{k}(1+t)^{n-k} .
\end{gathered}
$$

Отсюда следует, что

$$
\sum_{m}\left(\begin{array}{l}
n \\
m
\end{array}\right) P_{k}(m ; n) t^{m}=\left(\begin{array}{l}
n \\
k
\end{array}\right)(1-t)^{k}(1+t)^{n-k} .
$$

Полагая здесь $t=1$, получаем при $k>0$ соотношение

$$
\sum_{m}\left(\begin{array}{l}
n \\
m
\end{array}\right) P_{k}(m ; n)=0
$$

(аналог соотношения (17)); отметим также, что $P_{0}(m ; n)=1$ для всех $m \geq 0$. 
2) Имеют место следующие интегральные представления: при чётном $m$

$$
P_{k}(m ; n)=(-1)^{\frac{m}{2}} \frac{2^{n+1}}{\pi} \int_{0}^{\pi / 2} \cos [(n-2 k) x] \sin ^{m} x \cos ^{n-m} x \mathrm{~d} x,
$$

при нечётном $m$

$$
P_{k}(m ; n)=(-1)^{\frac{m-1}{2}} \frac{2^{n+1}}{\pi} \int_{0}^{\pi / 2} \sin [(n-2 k) x] \sin ^{m} x \cos ^{n-m} x \mathrm{~d} x .
$$

Действительно, из (16) следует, что

$$
\begin{gathered}
P_{k}(m ; n)=\frac{1}{2 \pi i} \int_{|z|=1} \frac{(1-z)^{m}(1+z)^{n-m}}{z^{k+1}} \mathrm{~d} z= \\
=\frac{1}{2 \pi} \int_{-\pi}^{\pi} e^{-i k \phi}\left(1-e^{i \phi}\right)^{m}\left(1+e^{i \phi}\right)^{n-m} \mathrm{~d} \phi .
\end{gathered}
$$

Ho

$$
1-e^{i \phi}=-2 i \sin \frac{\phi}{2} e^{i \frac{\phi}{2}}, \quad 1+e^{i \phi}=2 \cos \frac{\phi}{2} e^{i \frac{\phi}{2}}
$$

следовательно,

$$
\begin{gathered}
P_{k}(m ; n)=\frac{(-i)^{m} 2^{n}}{2 \pi} \int_{-\pi}^{\pi} e^{i \frac{\phi}{2}(n-2 k)} \sin ^{m} \frac{\phi}{2} \cos ^{n-m} \frac{\phi}{2} \mathrm{~d} \phi= \\
=\frac{(-i)^{m} 2^{n}}{2 \pi} \int_{0}^{\pi} \sin ^{m} \frac{\phi}{2} \cos ^{n-m} \frac{\phi}{2}\left[e^{i(n-2 k) \frac{\phi}{2}}+(-1)^{m} e^{-i(n-2 k) \frac{\phi}{2}}\right] \mathrm{d} \phi,
\end{gathered}
$$

откуда, в зависимости от чётности $m$, получаем указанные формулы.

Из указанных интегральных представлений, в частности, следует, что при нечётном $m$

$$
P_{n}(m ; 2 n)=0
$$

а при чётном $m$

$$
\begin{aligned}
& P_{n}(m ; 2 n)=(-1)^{\frac{m}{2}} \frac{2^{2 n+1}}{\pi} \int_{0}^{\pi / 2} \sin ^{m} x \cos ^{2 n-m} x \mathrm{~d} x= \\
& =(-1)^{\frac{m}{2}} \frac{2^{2 n}}{\pi} \mathrm{B}\left(\frac{m+1}{2}, \frac{2 n-m+1}{2}\right),
\end{aligned}
$$

где $\mathrm{B}(a, b)$ - бета-функция: $\mathrm{B}(a, b)=\int_{0}^{1} t^{a-1}(1-t)^{b-1} \mathrm{~d} t, a>0, b>0$. 
Из этих представлений непосредственно следует также общая оценка

$$
\left|P_{k}(m ; n)\right| \leq \frac{2^{n+1}}{\pi} \int_{0}^{\pi / 2} \sin ^{m} x \cos ^{n-m} x \mathrm{~d} x=\frac{2^{n}}{\pi} \mathrm{B}\left(\frac{m+1}{2}, \frac{n-m+1}{2}\right) .
$$

Подчеркнём, что эта оценка равномерна по $k$.

Известно [7], что функция $\mathrm{B}(x)=\mathrm{B}(x, a-x), 0<x<a$, монотонно убывает на интервале $(0, a / 2)$ и монотонно возрастает на интервале $(a / 2, a)$, поэтому правая часть в (22) как функция $m$ монотонно убывает на интервале от 0 до $\left[\frac{n}{4}+\frac{1}{2}\right]$, а далее монотонно возрастает. В крайних точках имеем оценки

$$
\left|P_{k}(0 ; n)\right|=\left|P_{k}(n ; n)\right| \leq \frac{2^{n}}{\pi} \mathrm{B}\left(\frac{1}{2}, \frac{n+1}{2}\right)=2^{n} \frac{\Gamma\left(\frac{n+1}{2}\right)}{\Gamma\left(\frac{n+2}{2}\right)} \leq 2^{n} \sqrt{\frac{2}{n}}\left(1-\frac{1}{n+1}\right),
$$

и это есть оценка максимума $P_{k}(m ; n)$ при всех значениях $k$.

3) С помощью производящей функции (16) находим:

$$
\begin{gathered}
\sum_{k=0}^{n} P_{k}(l ; n) P_{k}(m ; n)=\left[z^{0}\right] P(z ; l, n) P\left(\frac{1}{z} ; m, n\right)= \\
=\left[z^{0}\right](1-z)^{l}(1+z)^{n-l}\left(1-\frac{1}{z}\right)^{m}\left(1+\frac{1}{z}\right)^{n-m}= \\
=(-1)^{m}\left[z^{n}\right](1-z)^{l+m}(1+z)^{2 n-l-m}= \\
=(-1)^{m} P_{n}(l+m ; 2 n)=(-1)^{l} P_{n}(l+m ; 2 n)=\sigma_{l m}(n) .
\end{gathered}
$$

Это с учётом (20) и (21) даёт следующий результат:

$$
\sigma_{l m}(n)=\left\{\begin{array}{cc}
0, & \text { если } l+m \text { нечётно, } \\
(-1)^{m+\frac{l+m}{2}} \frac{2^{2 n}}{\pi} \mathrm{B}\left(\frac{l+m+1}{2}, n-\frac{l+m-1}{2}\right), & \text { если } l+m \text { чётно. }
\end{array}\right.
$$

Отметим важный частный случай этого результата:

$$
\sigma_{m m}(n)=\sigma_{m}^{2}(n)=\sum_{k=0}^{n} P_{k}^{2}(m ; n)=\frac{2^{2 n}}{\pi} \mathrm{B}\left(m+\frac{1}{2}, n-m+\frac{1}{2}\right),
$$

при этом $\sigma_{n-m}^{2}(n)=\sigma_{m}^{2}(n)$. 
Используя асимптотическую формулу Стирлинга для гамма-функции

$$
\Gamma(x+1) \sim x^{x} e^{-x} \sqrt{2 \pi x}, \quad x \rightarrow \infty,
$$

из (24) нетрудно получить следующие асимптотические оценки (они нам понадобятся в дальнейшем):

$$
\sigma_{m}^{2}(n) \sim \frac{2^{2 n}}{\pi} \Gamma\left(m+\frac{1}{2}\right) n^{-m-\frac{1}{2}}
$$

если $n \rightarrow \infty$, а $m \geq 0$ фиксировано;

$$
\sigma_{m}^{2}(n) \sim \sqrt{\frac{2}{\pi n}} 2^{2 n} m^{m}(n-m)^{n-m} n^{-n},
$$

если $n, m, n-m \rightarrow \infty$.

В дальнейшем нам понадобится также следующая формула для суммы лишь по чётным значениям индекса $k$ :

$$
\sum_{k \geq 0} P_{2 k}^{2}(m ; n)=\frac{2^{2 n-1}}{\pi} \mathrm{B}\left(m+\frac{1}{2}, n-m+\frac{1}{2}\right)+\frac{(-1)^{m+n / 2}}{2}\left(\begin{array}{c}
n \\
n / 2
\end{array}\right) I(n \equiv 0(\bmod 2))
$$

(сумма по нечётным значениям индекса $k$ получается вычитанием этого выражения из (24)). Подчеркнём, что при нечётном $n$ эта сумма равна половине полной суммы (24).

Формула (27) доказывается аналогично (24), исходя из соотношений (см. (16))

$$
\begin{aligned}
& P(z ; m, n)+P(-z ; m, n)=2 \sum_{k \geq 0} P_{2 k}(m ; n) z^{k}= \\
= & (1-z)^{m}(1+z)^{n-m}+(1+z)^{m}(1-z)^{n-m}=Q(z)
\end{aligned}
$$

и

$$
\sum_{k \geq 0} P_{2 k}^{2}(m ; n)=\left[z^{0}\right] Q(z) Q\left(\frac{1}{z}\right)
$$

4) Отметим дополнительно некоторые свойства матрицы Кравчука $P_{n}=\left(P_{k}(m ; n)\right)_{k, m=0,1, \ldots, n}$, определённой в (7). Первая её строка состоит целиком из единиц. Сумма всех строк составляет вектор $\left(2^{n}, 0, \ldots, 0\right)$. Первый столбец 
состоит из биномиальных коэффициентов: $\left(\left(\begin{array}{l}n \\ 0\end{array}\right),\left(\begin{array}{l}n \\ 1\end{array}\right), \ldots,\left(\begin{array}{l}n \\ n\end{array}\right)\right)^{\prime}$. Симметричные относительно главной диагонали элементы связаны соотношением

$$
\left(\begin{array}{l}
n \\
m
\end{array}\right) P_{k}(m ; n)=\left(\begin{array}{l}
n \\
k
\end{array}\right) P_{m}(k ; n), 0 \leq k, m \leq n,
$$

то есть матрица не является симметрической, в то же время нормированная матрица с элементами $\left(\begin{array}{l}n \\ k\end{array}\right)^{-1} P_{k}(m ; n)$ свойством симметрии обладает.

Отметим также, что нулевые элементы матрицы расположены симметрично относительно главной диагонали.

При чётных значениях $n$ средний столбец (с номером $1+n / 2)$ состоит из элементов $(-1)^{k / 2}\left(\begin{array}{l}n / 2 \\ k / 2\end{array}\right)$ при чётных $k$, а при нечётных $k$ эти элементы равны 0. Аналогичное свойство имеет место и для средней строки.

$$
\begin{aligned}
& \text { Примеры. } P_{2}=\left(\begin{array}{ccc}
1 & 1 & 1 \\
2 & 0 & -2 \\
1 & -1 & 1
\end{array}\right), \quad P_{2} P_{2}=\left(\begin{array}{lll}
4 & 0 & 0 \\
0 & 4 & 0 \\
0 & 0 & 4
\end{array}\right)=2^{2} I_{3} \text {, } \\
& P_{3}=\left(\begin{array}{cccc}
1 & 1 & 1 & 1 \\
3 & 1 & -1 & -3 \\
3 & -1 & -1 & 3 \\
1 & -1 & 1 & -1
\end{array}\right), \quad P_{3}^{2}=2^{3} I_{4}
\end{aligned}
$$

5) Наконец, отметим связь многочленов Кравчука с широко известной в анализе гипергеометрической функцией $F(a ; b ; c ; z)$ [4]:

$$
F(a ; b ; c ; z)=1+\sum_{k \geq 1}\left[\prod_{j=0}^{k-1} \frac{(a+j)(b+j)}{(c+j)}\right] \frac{z^{k}}{k !},|z|<1 .
$$

Пусть случайная величина $\mathrm{X}$ имеет гипергеометрическое распределение с параметрами $m, n$ и $k$ :

$$
\mathbf{P}\{X=x\}=\frac{\left(\begin{array}{c}
m \\
x
\end{array}\right)\left(\begin{array}{l}
n-m \\
k-x
\end{array}\right)}{\left(\begin{array}{l}
n \\
k
\end{array}\right)}, 0 \leq x \leq \min (k, m) .
$$


Тогда

$$
P_{k}(m ; n)=2\left(\begin{array}{l}
n \\
k
\end{array}\right)\left(\mathrm{P}\{X \equiv 0(\bmod 2)\}-\frac{1}{2}\right),
$$

а также

$$
P_{k}(m ; n)=\left(\begin{array}{l}
n \\
k
\end{array}\right) F(-k ;-m ; n-m ;-1) .
$$

3. Моменты спектра симметрической функции. Общие формулы для первых и вторых моментов случайного спектра $\bar{w}_{n}^{f}=\left(w_{0}^{f}, w_{1}^{f}, \ldots, w_{n}^{f}\right)$ в $\bar{p}$-модели сразу следуют из представления (8):

$$
\begin{gathered}
\mathbf{E} \bar{w}_{n}^{f}=\mathbf{E} \bar{f}_{n} P_{n}, \\
\mathbf{D} \bar{w}_{n}^{f}=\left(\operatorname{cov}\left(w_{l}^{f}, w_{m}^{f}\right)\right)_{0}^{n}=P_{n}^{\prime} \mathbf{D} \bar{f}_{n} P_{n} .
\end{gathered}
$$

Здесь

$$
\begin{gathered}
\mathbf{E} \bar{f}_{n}=\bar{p}=\left(p_{0}, p_{1}, \ldots, p_{n}\right), \\
\mathbf{D} \bar{f}_{n}=\operatorname{Diag}\left(p_{0} q_{0}, p_{1} q_{1}, \ldots, p_{n} q_{n}\right),
\end{gathered}
$$

где $\operatorname{Diag}(\bar{a})$ - диагональная матрица с главной диагональю $\bar{a}$, поэтому формулы (28) в координатной записи принимают вид (далее для упрощения записи верхний индекс $f$ мы опускаем):

$$
\begin{gathered}
\mu_{m}(n)=\mathbf{E} w_{m}=\sum_{k=0}^{n} p_{k} P_{k}(m ; n), \quad m=0,1, \ldots, n, \\
d_{l m}(n)=\operatorname{cov}\left(w_{l}, w_{m}\right)=\sum_{k=0}^{n} p_{k} q_{k} P_{k}(l ; n) P_{k}(m ; n), \quad l, m=0,1, \ldots, n .
\end{gathered}
$$

В частном случае, когда все $p_{k}=p>0$ ( $p$-модель), формулы значительно упрощаются: из (17) следует, что

$$
\mu_{0}(n)=p 2^{n}, \quad \mu_{m}(n)=0, m>0,
$$

a (cM. (23))

$$
d_{l m}(n)=p q \sum_{k=0}^{n} P_{k}(l ; n) P_{k}(m ; n)=p q \sigma_{l m}(n) .
$$


Таким образом, компоненты спектра с номерами разной чётности в этом случае оказываются некоррелированными, а для дисперсий получаем представления (см. (24))

$$
d_{m}^{2}(n)=\mathbf{D} w_{m}=p q \sigma_{m}^{2}(n), \quad m=0,1, \ldots, n .
$$

Отметим также следующие свойства дисперсий в $p$-модели, являющиеся прямым следствием свойств бета-функции:

1) симметрия: $d_{m}^{2}(n)=d_{n-m}^{2}(n)$,

2) монотонность: $d_{m}^{2}(n)>d_{m+1}^{2}(n), 0 \leq m \leq \frac{n}{2}-1$.

Из приведённой в (25)-(26) асимптотики следует, что с ростом $m$ эти дисперсии весьма быстро убывают, достигая своего минимального значения, асимптотически равного $p q \sqrt{\frac{2}{\pi n}} 2^{n}$, при $m=\left[\frac{n}{2}\right]$.

4. Предельные распределения. В этом разделе мы обсудим вопросы асимптотического поведения при $n \rightarrow \infty$ различных характеристик спектра случайной симметрической булевой функции в общей $\bar{p}$-модели (см. введение). Как следует из представления (6), в рассматриваемой модели компоненты спектра представляют собой линейные комбинации независимых бернуллиевских случайных величин, поэтому следует ожидать, что к ним применима центральная предельная теорема теории вероятностей, а специфика модели связана с асимптотическими свойствами многочленов Кравчука. Инструментом же для получения соответствующих результатов будет служить нам общая характеристическая функция спектра (15).

1) Общая $\bar{p}$-модель. Введём нормированные случайные величины (см. (29)-(32))

$$
w_{m}^{*}=\frac{w_{m}-\mu_{m}(n)}{d_{m}(n)}, \quad m=0,1, \ldots, n,
$$

где

$$
d_{m}^{2}(n)=\mathbf{D} w_{m}=\sum_{k=0}^{n} p_{k} q_{k} P_{k}^{2}(m ; n),
$$

и будем предполагать выполненным условие

$$
0<c_{1} \leq p_{k} \leq c_{2}<1, \quad k=0,1, \ldots, n .
$$


Тогда $1-c_{2} \leq q_{k} \leq 1-c_{1}, \quad k=0,1, \ldots, n$, и для всех $k$ имеют место оценки

$$
0<c=\max \left(c_{1}\left(1-c_{2}\right), c_{2}\left(1-c_{1}\right)\right) \leq p_{k} q_{k} \leq \frac{1}{4},
$$

и для дисперсий $d_{m}^{2}(n)$ получаем неравенства

$$
c \sigma_{m}^{2}(n) \leq d_{m}^{2}(n) \leq \frac{1}{4} \sigma_{m}^{2}(n),
$$

из которых следует (см. (32)), что асимптотическое поведение дисперсий элементов спектра в $\bar{p}$-модели такое же, как и в $p$-модели.

Однако такого нельзя сказать о корреляциях. Рассмотрим, например, корреляцию симметричных величин $w_{m}$ и $w_{n-m}$. В силу свойства

$$
\begin{gathered}
P_{k}(m ; n)=(-1)^{k} P_{k}(n-m ; n) \text { (см. (16)) } \\
\rho_{m, n-m}(n)=\operatorname{corr}\left(w_{m}, w_{n-m}\right)=\sum_{k=0}^{n}(-1)^{k} p_{k} q_{k} P_{k}^{2}(m ; n) / d_{m}^{2}(n),
\end{gathered}
$$

и из (23)-(24) следует, что в $p$-модели этот коэффициент корреляции при нечётном $n$ равен нулю, а при чётном $n$

$$
\rho_{m, n-m}(n)=\sigma_{m, n-m}(n) / \sigma_{m}^{2}(n)=(-1)^{m+n / 2} \mathrm{~B}\left(\frac{n+1}{2}, \frac{n+1}{2}\right) / \mathrm{B}\left(m+\frac{1}{2}, n-m+\frac{1}{2}\right) .
$$

Применение формулы Стирлинга для гамма-функции даёт, что если $n \rightarrow \infty$, a $m \geq 0$ фиксировано, то эта корреляция убывает как $O\left(n^{m} 2^{-n}\right)$, то есть в этом случае рассматриваемые величины асимптотически не коррелированы.

Рассмотрим теперь ту же корреляцию (35) в следующем варианте $\bar{p}$-модели: $p_{k}=p_{1}$, если $k$ нечётно, и $p_{k}=p_{2}$, если $k$ чётно. Тогда

$$
\rho_{m, n-m}(n)=\frac{p_{2} q_{2} \sum_{k \geq 0} P_{2 k}^{2}(m ; n)-p_{1} q_{1} \sum_{k \geq 0} P_{2 k+1}^{2}(m ; n)}{p_{2} q_{2} \sum_{k \geq 0} P_{2 k}^{2}(m ; n)+p_{1} q_{1} \sum_{k \geq 0} P_{2 k+1}^{2}(m ; n)},
$$

и из (27) следует, что при тех же условиях, что и выше, этот коэффициент корреляции имеет пределом величину $\left(p_{2} q_{2}-p_{1} q_{1}\right) /\left(p_{2} q_{2}+p_{1} q_{1}\right)$, которая отлична от нуля, если $p_{1} \neq p_{2}$. Этот пример говорит о том, что свойство асимптотической некоррелированности спектральных коэффициентов симметрической булевой функции, имеющее место в $p$-модели, не выполняется, вообще говоря, в общей $\bar{p}$-модели. 
После этих предварительных рассмотрений перейдём к основному вопросу - анализу предельных распределений элементов спектра, и, прежде всего, установим следующий результат.

Теорема 1. Пусть выполнено условие (33) и для определённого в (35) коэффициента корреляции $\rho_{m, n-m}(n)$ nри $n \rightarrow \infty$ и фиксированном $m \geq 0$ существует предел $\lim \rho_{m, n-m}(n)=\rho$. Тогда совместное распределение величин $w_{m}$ и $w_{n-m}$ асимптотически нормально:

$$
\mathscr{L}\left(w_{m}^{*}, w_{n-m}^{*}\right) \sim \mathcal{N}\left((0,0),\left(\begin{array}{ll}
1 & \rho \\
\rho & 1
\end{array}\right)\right) .
$$

В частности, в р-модели эти величины также и асимптотически независимы.

Доказательство. Из общей характеристической функции спектра (15) имеем (напомним, что $d_{m}=d_{n-m}$, так как $P_{k}(m ; n)=(-1)^{k} P_{k}(n-m ; n)$, и для сокращения записи аргумент $n$ у $\mu_{m}$ и $d_{m}$ опускаем)

$$
\begin{gathered}
\mathbf{E} e^{i\left(t_{1} w_{m}^{*}+t_{2} w_{n-m}^{*}\right)}=\exp \left\{-i\left(t_{1} \mu_{m}+t_{2} \mu_{n-m}\right) / d_{m}\right\} \times \\
\times \prod_{k=0}^{n}\left(q_{k}+p_{k} \exp \left\{i\left(t_{1} P_{k}(m ; n)+t_{2} P_{k}(n-m ; n)\right) / d_{m}\right\}\right) .
\end{gathered}
$$

Из оценок (22), (25) и (34) с использованием известной связи бета- и гаммафункций, а также формулы Стирлинга для последней, следует, что в рассматриваемых условиях равномерно по $k$

$$
\left|P_{k}(m ; n)\right| / d_{m}=O\left(n^{-1 / 4}\right),
$$

поэтому можем записать следующую цепочку соотношений:

$$
\begin{gathered}
\ln \mathbf{E} e^{i\left(t_{1} w_{m}^{*}+t_{2} w_{n-m}^{*}\right)}=-i\left(t_{1} \mu_{m}+t_{2} \mu_{n-m}\right) / d_{m}+ \\
+\sum_{k=0}^{n} \ln \left(1+p_{k}\left(\exp \left\{i\left(t_{1}+(-1)^{k} t_{2}\right) P_{k}(m ; n) / d_{m}\right\}-1\right)\right)= \\
=-i\left(t_{1} \mu_{m}+t_{2} \mu_{n-m}\right) / d_{m}+\sum_{k=0}^{n}\left(p_{k}\left(\exp \left\{i\left(t_{1}+(-1)^{k} t_{2}\right) P_{k}(m ; n) / d_{m}\right\}-1\right)\right)- \\
-\frac{1}{2} \sum_{k=0}^{n}\left(p_{k}\left(\exp \left\{i\left(t_{1}+(-1)^{k} t_{2}\right) P_{k}(m ; n) / d_{m}\right\}-1\right)\right)^{2}+O\left(\sum_{k=0}^{n}\left|P_{k}(m ; n)\right|^{3} d_{m}^{-3}\right)=
\end{gathered}
$$




$$
\begin{gathered}
=-\frac{1}{2} \sum_{k=0}^{n} p_{k}\left(\left(t_{1}+(-1)^{k} t_{2}\right) P_{k}(m ; n) / d_{m}\right)^{2}+ \\
+\frac{1}{2} \sum_{k=0}^{n} p_{k}^{2}\left(\left(t_{1}+(-1)^{k} t_{2}\right) P_{k}(m ; n) / d_{m}\right)^{2}+O\left(n^{-1 / 4}\right)= \\
=-\frac{1}{2}\left(t_{1}^{2}+2 t_{1} t_{2} \rho_{m, n-m}(n)+t_{2}^{2}\right)+O\left(n^{-1 / 4}\right) .
\end{gathered}
$$

Здесь все остаточные члены разложений имеют вид $O\left(\sum_{k=0}^{n}\left|P_{k}(m ; n)\right|^{3} d_{m}^{-3}\right)$. Вынося за знак суммы максимум из $\left|P_{k}(m ; n)\right| / d_{m}$, имеющий порядок $O\left(n^{-1 / 4}\right)$, получим сумму квадратов, которая в силу соотношений (24), (34) имеет порядок $O(1)$. Отсюда следует вид остатка.

В условиях теоремы это выражение имеет пределом $-\frac{1}{2}\left(t_{1}^{2}+2 t_{1} t_{2} \rho+t_{2}^{2}\right)$, что и требовалось доказать.

Аналогично устанавливается и асимптотическая нормальность произвольного конечного набора $\bar{w}^{*}(n)=\left(w_{m_{1}}^{*}, \ldots, w_{m_{s}}^{*}\right), \quad 0 \leq m_{1}<\ldots<m_{s}$, спектральных коэффициентов.

Теорема 2. Пусть выполнено условие (33) и для коэффициентов коррелящии $\rho_{l m}(n)=d_{l m}(n) /\left(d_{l}(n) d_{m}(n)\right)$ (см. (29)) при $n \rightarrow \infty \quad$ и любых фиксированных $l$ и $m$ существуют предель $\lim \rho_{l m}(n)=\rho_{l m}$. Тогда вектор $\bar{w}^{*}(n)=\left(w_{m_{1}}^{*}, \ldots, w_{m_{s}}^{*}\right) \quad$ при любых фиксированных $0 \leq m_{1}<\ldots<m_{s}$ асилптотически нормален с нулевым математическим ожиданием иматрицей вторых моментов $\left(\rho_{m_{i} m_{j}}\right)_{i, j=1, \ldots, s}$.

Для доказательства достаточно установить, что характеристическая функция

$$
\operatorname{Eexp}\left\{i \sum_{j=1}^{s} t_{j} w_{m_{j}}^{*}\right\}=\exp \left\{-i \sum_{j=1}^{s} t_{j} \frac{\mu_{m_{j}}}{d_{m_{j}}}\right\} \prod_{k=0}^{n}\left(1+p_{k}\left(\exp \left\{i \sum_{j=1}^{s} t_{j} \frac{P_{k}\left(m_{j} ; n\right)}{d_{m_{j}}}\right\}-1\right)\right)
$$

в указанных условиях имеет пределом величину $\exp \left\{-\frac{1}{2} \sum_{i, j=1}^{s} t_{i} t_{j} \rho_{m_{i} m_{j}}\right\}$, что обосновывается аналогично доказательству теоремы 1. 
Замечание. В случае $p$-модели для коэффициентов корреляции имеем представления (см. (23)-(24))

$$
\rho_{l m}(n)=(-1)^{l+\frac{l+m}{2}} \frac{\mathrm{B}\left(\frac{l+m+1}{2}, n-\frac{l+m-1}{2}\right)}{\sqrt{\mathrm{B}\left(l+\frac{1}{2}, n-l+\frac{1}{2}\right) \mathrm{B}\left(m+\frac{1}{2}, n-m+\frac{1}{2}\right)}}
$$

(если $l+m$ чётно), а применение здесь формулы Стирлинга для гаммафункции даёт, что в рассматриваемых условиях эти величины имеют пределы

$$
\lim \rho_{l m}(n)=\rho_{l m}=(-1)^{l+\frac{l+m}{2}} \Gamma\left(\frac{l+m+1}{2}\right) / \sqrt{\Gamma\left(l+\frac{1}{2}\right) \Gamma\left(m+\frac{1}{2}\right)} .
$$

Подчеркнём следующую особенность этого результата. Как следует из соотношений (34) и (25), при $n \rightarrow \infty$ и ограниченных значениях $m$ дисперсии $d_{m}^{2}(n)=\mathbf{D} w_{m}$ весьма быстро убывают с увеличением $m$ : $d_{m+1}(n) / d_{m}(n)=O\left(n^{-1 / 2}\right)$ (напомним, что $d_{m}=d_{n-m}$ ), поэтому компоненты вектора $\bar{w}^{*}(n)=\left(w_{m_{1}}^{*}, \ldots, w_{m_{s}}^{*}\right)$ имеют разный порядок нормировки. Отсюда следует, в частности, что спектральные коэффициенты весьма сильно «разделены»: с большой вероятностью максимальными являются крайние из них. Например, в условиях теоремы 2

$$
\mathbf{P}\left(\left|w_{m+1}-\mu_{m+1}\right|<\left|w_{m}-\mu_{m}\right|\right)=\mathbf{P}\left(\left|w_{m+1}^{*}\right|<\left|w_{m}^{*}\right| \frac{d_{m}}{d_{m+1}}\right) \rightarrow 1,
$$

поскольку множитель $\frac{d_{m}}{d_{m+1}}$ неограниченно возрастает, а случайные величины $w_{m}^{*}$ и $w_{m+1}^{*}$ имеют собственные предельные распределения.

Более детально эти важные для приложений вопросы мы обсудим в рамках аналитически более просто устроенной $p$-модели.

2) Симметричная p-модель. В этом случае (см. (30)-(32)) выражения для моментов спектральных коэффициентов значительно упрощаются, что позволяет осуществить дальнейшее продвижение в анализе асимптотических свойств спектра. 
Будем под $w_{0}$ понимать далее центрированную величину $w_{0}-p 2^{n}$ (остальные спектральные коэффициенты в рассматриваемом случае центрированы в нуле) и рассмотрим следующую характеристику спектра:

$$
\Delta_{m}(n)=\max _{m \leq k \leq n-m}\left|w_{k}\right| / d_{m}, m \geq 0 .
$$

Имеет место следующее утверждение.

Теорема 3. $B$ р-модели при $n \rightarrow \infty$ и любом фиксированном $m \geq 0$ случайная величина (36) имеет предельную функцию распределения $(2 \Phi(x)-1)^{2}, x>0$, где $\Phi(x)$ - функиия стандартного нормального распределения.

Доказательство. Как следует из теоремы 1 , при $n \rightarrow \infty$ и, ограниченных значениях $m$ предельное распределение величины

$$
\delta_{m}(n)=\max \left(\left|w_{m}^{*}\right|,\left|w_{n-m}^{*}\right|\right)
$$

существует:

$$
\mathbf{P}\left(\delta_{m}(n)<x\right)=\mathbf{P}\left(\left|w_{m}^{*}\right|<x,\left|w_{n-m}^{*}\right|<x\right) \rightarrow(2 \Phi(x)-1)^{2}, x>0 .
$$

Такое же предельное распределение в этих условиях имеет и определённая в (36) характеристика. Чтобы установить это, достаточно показать, что величина

$$
\Delta_{m}^{\prime}(n)=\max _{m+1 \leq k \leq n-m-1}\left|w_{k}\right| / d_{m}
$$

стремится по вероятности к нулю. Используя неравенство Чебышева, имеем

$$
\mathbf{P}\left(\Delta_{m}^{\prime}(n)>\varepsilon\right)=\mathbf{P}\left(\bigcup_{k=m+1}^{n-m-1}\left\{\left|w_{k}\right|>\varepsilon d_{m}\right\}\right) \leq \sum_{k=m+1}^{n-m-1} \mathbf{P}\left(\left|w_{k}\right|>\varepsilon d_{m}\right) \leq \sum_{k=m+1}^{n-m-1} \frac{d_{k}^{2}}{\varepsilon^{2} d_{m}^{2}} .
$$

Из (25) и (26), а также свойства монотонности дисперсий следует, что $d_{k}^{2} / d_{m}^{2}=O\left(n^{-1}\right) \quad$ при $k=m+1 \quad$ и $k=n-m-1 \quad$ и $\quad d_{k}^{2} / d_{m}^{2}=O\left(n^{-2}\right) \quad$ при $m+2 \leq k \leq n-m-2$. Поэтому вся сумма в правой части (37) есть величина $O\left(n^{-1}\right)$, что и требовалось показать.

Замечание. Аналогичными рассуждениями можно также установить, что если вместо характеристики (36) рассмотреть характеристику

$$
\Delta_{m, s}(n)=\max _{m \leq k \leq n-s}\left|w_{k}\right| / d_{\min (m, s)}, m \neq s,
$$

то при любых фиксированных $m$ и $s$ она имеет предельную функцию распределения $2 \Phi(x)-1, x>0$, такую же, как и случайная величина $\left|w_{\min (m, s)}^{*}\right| \cdot$ 
Эти результаты позволяют заключить, что в $p$-модели с вероятностью, стремящейся к 1, максимальными среди всех (либо части) спектральных коэффициентов являются крайние из них. Если же говорить более конкретно, то структура спектра $\bar{w}_{n}=\left(w_{0}, w_{1}, \ldots, w_{n}\right)$ случайной симметрической булевой функции устроена асимптотически следующим образом.

Максимальным коэффициентом является $w_{0}$ : он принимает значения в окрестности точки $p 2^{n}$, и размер этой окрестности имеет порядок $O\left(2^{n} / h^{1 / 4}\right)$; все остальные коэффициенты принимают значения в окрестностях нуля, причём размеры соответствующих окрестностей резко различаются: для $w_{n}$ разброс имеет порядок $O\left(2^{n} / n^{1 / 4}\right)$, для $w_{1}$ и $w_{n-1}-$ порядок $O\left(2^{n} / n^{3 / 4}\right)$, для $w_{2}$ и $w_{n-2}$ - порядок $O\left(2^{n} / n^{5 / 4}\right)$, и так далее.

\section{Список литературы}

1. Canteaut A., Videau M. Symmetric Boolean Function // IEEE Trans. Inf. Theory. - 2005. - V. 51. № 8. - P. 2791-2811.

2. Sarkar S., Maitra S. Efficient search for symmetric Boolean functions under constraints on Walsh spectra values // J. Comb. Math. and Comb. Comput. 2009. - V. 68. - P. 163-191.

3. Mouffron M. Balanced alternating and symmetric functions over finite sets // Workshop on Boolean Functions: Cryptography and Applications (BFCA'08). - 2008. - Copenhagen, Denmark. - P. 27-44.

4. Сегё Г. Ортогональные многочлены. - М.: ФИЗМАТЛИТ, 1962.

5. Мак-Вильямс Ф. Дж., Слоэн Н. Дж. А. Теория кодов, исправляющих ошибки. - М.: Связь, 1979.

6. Сидельников В. М. Теория кодирования. - М.: ФИЗМАТЛИТ, 2008.

7. Бэйтмен $Г$., Эрдейи А. Высшие трансцендентные функции. - М.: Наука, 1965.

8. Cusick Th. W., Stanica P. Cryptographic Boolean Functions and Applications // AP Elsevier, Amsterdam etc. — 2009.

9. Savicky P. On the bent Boolean functions that are symmetric // European J. Comb. - 1994. - V. 15. - P. 407-410.

10. Хохлов В.И. Многочлены, ортогональные относительно полиномиального распределения, и факториально-степенной формализм // Теория вероятности и её применение. - 2001. - Т. 46. Вып. 3. С. 585-592.

11. Хохлов В. И. Многочлены Эрмита и многочлены Пуассона-Шарлье как классические пределы многочленов Кравчука // Обозр. прикл. и промышл. матем. — 2005. — Т. 16. Вып. 5. — С. 937-940. 